\title{
OBSERVATIONAL EVIDENCE FOR SUPERMASSIVE BLACK HOLES
}

\author{
Alan Dressler \\ Mount Wilson and Las Campanas Observatories \\ Carnegie Institution of Washington \\ 813 Santa Barbara Street \\ Pasadena, CA 91101 \\ USA
}

ABSTRACT. A growing body of evidence from stellar dynamics in the nuclei of galaxies indicates that supermassive black holes of $10^{7}-10^{9} \quad M_{0}$ are common. The two best cases are M31 and M32, for which dark, central mass concentrations are the only straightforward interpretation. M87 continues to be a possible location of an even more massive black hole, but new observations and models by the author and $D$. Richstone effectively rule out the high black hole mass $\sim 5 x$ $10^{9} M_{0}$ claimed by Sargent, Young, and collaborators. New data are available for several other nearby galaxies which also show kinematic signatures that could also be due to supermassive black holes. The Hubble Space Telescope will play the key role in strengthening these cases and eliminating, for the best examples, alternative models which do not require supermassive black holes.

\section{INTRODUCTION}

The notion that a supermassive black hole is the crucial component of AGN powerhouse has been around for a long time. In 1969, Lynden-Bell listed the quasar properties that drove theorists of the time to conclude that the energy of quasars is probably gravitational in origin and most likely due to accretion onto a supermassive black hole. The crucial evidence comes from rapid changes in luminosity (about a day) which imply a size no bigger than the solar system. Even if the energy output could be supplied by thermonuclear processes with an efficiency of about $1 \%, \sim 10^{9} M_{0}$ are required for an average luminosity quasar. However, $10^{9} \mathrm{M}_{0}$ packed into a volume the size of the solar system has a gravitational binding energy comparable to or greater than the nuclear energy source. As Rees (1984, see Fig. 1) points out, whether the mass is in the form of a supermassive star, a dense star cluster, or a black hole, the probable end state in any case is a supermassive black hole. Accretion onto holes with masses in the range $10^{7}-10^{9}$ match the basic requirements of energy and variability; Lynden-Bell noted that the accretion was likely to 
proceed through a viscous disk, an idea that has been elaborately developed in recent years.

What are the observable consequences of such a model, other than the AGN activity itself? Since the central densities in galactic nuclei seem to top out at around $1000 \mathrm{M}_{0}$ per $\mathrm{pc}^{3}$, supermassive black holes should dominate structure and kinematics for $R \lesssim 10 \mathrm{pc}$.

Therefore, observations of the light distributions, and the kinematics of gas and stars, should unmask them. This sounds simple enough, but there is still the question of where to look, since we do not yet know if AGN activity was a brief episode in the life of most galaxies, or a ongoing cataclysm in a few, rare objects. Lynden-Bell (1969) presented this issue very clearly. If one assumes that the quasar lifetime was a small fraction of the quasar evolution timescale (106 vs. $10^{9}$ years, for example), a simple evaluation of the quasar density implies that "dead quasars", i.e., dormant black holes, should be fairly common in the Local Supercluster - even a descendant of a rare radio-loud quasar should be present. On the other hand, if quasars had a long lifetime comparable to the evolution time, either in a continuous or bursting mode, very few galaxies would have been one-time quasars and therefore they should be harder to find. Thus, from the point of view of an observer, there are two possible strategies: (1) concentrate on AGNs in the belief that the activity betrays the presence of a supermassive black hole; (2) investigate typical galaxies which are more easily studied because they are less distant and their spectra are free of the complex AGN features.

I will briefly review progress along both these avenues, concentrating on evidence from stellar dynamics in the cores of galaxies. First I will discuss evidence for a supermassive black hole in the AGN M87, which has been a widely discussed example for 10 years. I'll present new data which, I hope, settles some of the controversial issues. I will conclude that there is still no substantial evidence for a supermassive black hole; one of $\delta 10^{9} M_{0}$ is still very much a possibility, although there is no kinematic evidence that compels it. The original suggestion of a hole much more massive than this requires a rather contrived model. I will also review what I think is persuasive, nearly compelling, evidence for less-monstrous black holes in M31 and M32. Here the situation is reversed: rather contrived models are necessary to avoid the conclusion that a supermassive black hole is present. If plausibility arguments leave you cold, content yourself that the launch of the HST will likely take some of these from the "probable" category to "certain". The goal of our twenty year search could be in sight.

\section{M87: THE STORY CONTINUES}

M87, a giant elliptical galaxy located in the core of the Virgo cluster, is a prime candidate for an aging quasar: its nucleus is a radio and $X$-ray source, and from it emanates an optical jet. It should have come as no surprise, then, that two papers by Young, Sargent, Boksenberg, and collaborators in 1978 reported probable detection of a 
supermassive black hole of 3-5 $310^{9} M_{0}$ in M87. Yet, in my recollection, at least, it was a surprise, possibly because of the boldness of the claim considering that it was rather model-dependent.

The evidence came in two forms. First, Young et al. (1978) demonstrated that the light distribution of M87 departs from a King model, peaking-up in the central few arcseconds. This shoulder (not to be confused with a unresolved central luminosity spike) is reminiscent of the cusp that would develop in the density distribution if an extremely massive object occupied the very center of the galaxy. The projected density in such a cusp falls as the $7 / 4$ power of the radius for a thermally relaxed black hole, or as the $3 / 2$ power for the case of adiabatic accretion (Young (1980a). Neither of these are steep enough to account for the central spike in M87, but could account for the residual shoulder in the light distribution after the central spike is removed.

Sargent et al. (1978) presented kinematic data which also suggested the presence of a central mass concentration M87. The velocity dispersion rises steadily from $\sigma \sim 300 \mathrm{~km} / \mathrm{sec}$ at $r=10$ " to $\sigma \sim 360 \mathrm{~km} / \mathrm{sec}$ at $r=1 "$. In Sargent et al. both the photometric and kinematic anomalies were explained by the presence of a 3-5 $\times 10^{9}$ solar mass condensed object, given the generic label "black hole". In a later paper, Young (1980b) emphasized that M87 was one of the very few systems known, at the time, to show a peaking of the light distribution within the core, and the only one known to have a rising velocity dispersion within the core. He admitted, however, that M87 was one of the only galaxies known to have such a well-resolved core $\left(r_{a} \sim 10 "\right)$, so few galaxies were available for comparison.

The supermassive black hole interpretation for M87 was challenged from two directions. Papers were published which criticized the assumption that the velocity distribution function in the core of M87 is isotropic. Duncan and Wheeler (1980), and later Binney and Mamon (1982) claimed that models with constant mass-to-light ratios (M/L) could be constructed if the distributions were made sufficiently anisotropic. Whether these models are physically reasonable has remained a controversial topic for many years. I will return to this point later.

The other challenge came from me, and it was an observational one. The model presented in Sargent et al. predicted a continuing rise in the stellar velocity dispersion within their last measured point, a radius of about 1.5 arcseconds. I obtained a spectrum of M87 from Las Campanas Observatory through a 1" $x$ 1" aperture in February, 1980 on a night of sub-arcsecond seeing. From analysis of this spectrum (Dressler 1980) I measured a velocity dispersion of $\sigma=350 \pm 32$ $\mathrm{km} / \mathrm{sec}$ at an effective radius of $r \sim 0.3$ arcseconds. This value was much lower than the $\sigma \sim 500 \mathrm{~km} / \mathrm{sec}$ predicted by the Sargent et al. model which included a supermassive black hole of $5 \times 10^{9} M_{0}$.

I also concluded that most of the light in the central spike was stellar rather than non-thermal, and thus proposed that a star cluster inhabited the core. Both of these claims have been challenged, most recently by Filippenko (1988), who correctly points out that atmospheric dispersion makes it impossible to keep all of the central 
spike and its underlying stars within a 1" $\times 1$ " aperture over the wavelength range $4000 \AA<\lambda<6000 \AA$. He and I have argued over the years about what was the likely configuration of my observation, and last year we concluded from a number of factors that I must have been approximately half-on, and half-off the nucleus in the crucial wavelength region $5000 \AA<\lambda<6000 \AA$. I am now able to report that this conclusion was essentially correct. I have new measurements taken with a long slit oriented along the atmospheric dispersion. The seeing during 2 of the 3 exposures of $2000 \mathrm{~s}$ was 1.0 arcseconds FWHM; this was verified by taking test exposures of a nearby star. These spectra (Fig. 1) are of much higher signal-to-noise ratio than my previous data, and, to my knowledge, are the best now available.

As a result of the analysis of these data, I have one confirmation and one retraction to make. The confirmation is that the velocity dispersion does not continue to rise but levels off at about $360 \mathrm{~km} / \mathrm{sec}$, as I had claimed. Fig. 2 shows that the dispersion is level for $r<2 "$. For the central 1.08" $\times 0.72^{\prime \prime}$ the measured dispersion is $360 \pm 10 \mathrm{~km} / \mathrm{sec}$. The dispersion falls for $r>2 "$, reaching $\sim 300 \mathrm{~km} / \mathrm{sec}$ at $10^{\prime \prime}$, in agreement with the data of Sargent et al. There is a feature in the rotation curve at the $50 \mathrm{~km} / \mathrm{sec}$ level that may or may not be real, but it is clear that $V_{\max }<100$
$\mathrm{~km} / \mathrm{sec}$ for the core.

In contradiction to my 1980 report, I now find that the central spike is mostly non-thermal, as Young et al. claimed. The Fourier analysis program solves for a line strength parameter, which can be clearly seen in Fig. 2 to drop for $r<1$ ". Assuming that the line-strengths of the stars remain constant, I calculate the dilution and find that it is consistent with the entire spike coming from a non-thermal source (Fig. 3).

Kormendy (1988a) has also obtained high spatial resolution spectra of M87 with the CFHT. With his lower S/N spectra he has not attempted to measure kinematics, but he comes to the same conclusion regarding the non-thermal nature of the central spike.

To discuss the consequences of these new observations, I will return to the question of whether the core of M87 can be modeled with a constant $M / L$. Richstone and Tremaine have developed the most general approach for modeling such systems, using a linear programming technique originally devised by Schwarzshild. They assemble a galaxy from a library of stellar orbits in such a way as to reproduce the kinematic data while simultaneously matching the density distribution, which is derived from the luminosity profile assuming some function $M / L=f(r)$. For each solution a distribution function of the stellar energy and angular momentum is determined. The predictions are given in discrete radial velocity bins, less appealing aesthetically than continuous functions, but more practical because the sampling of data and models can be exactly matched. A complete description of the method is given by Richstone and Tremaine (1984, 1985), including a comparison with another approach by Newton and Binney (1984) which is complementary.

In their 1985 paper, Richstone and Tremaine reached the same conclusion as earlier workers that a constant $M / L$ model for M87 was 


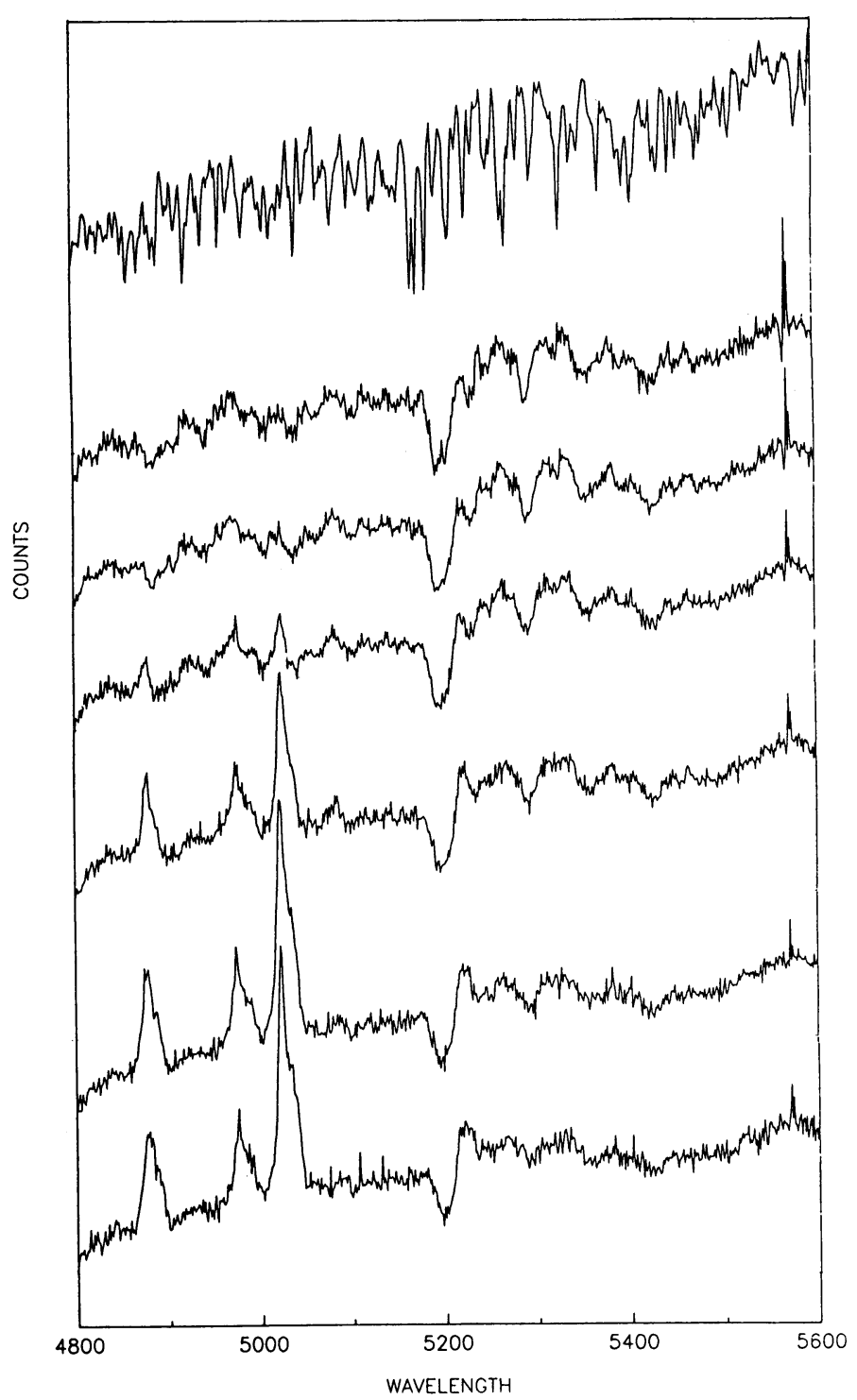

Figure 1. Spectra of a KOIII star (top) and, starting with the second from the top, spectra of M87 at effective radii of 8.5, 4.2, 2.0, 0.9, 0.4 , and 0.1 arcseconds from the nucleus. The increasing contamination of the spectra by non-thermal light, as traced by [0 III] and $\mathrm{H} \beta$ emission, is obvious. The $\mathrm{Mg}$ ' $\mathrm{b}$ ' triplet is seen clearly in all spectra, but $[\mathrm{N} \mathrm{I}] \lambda 5200 \AA$ from the non-thermal component contaminates the red wing. 


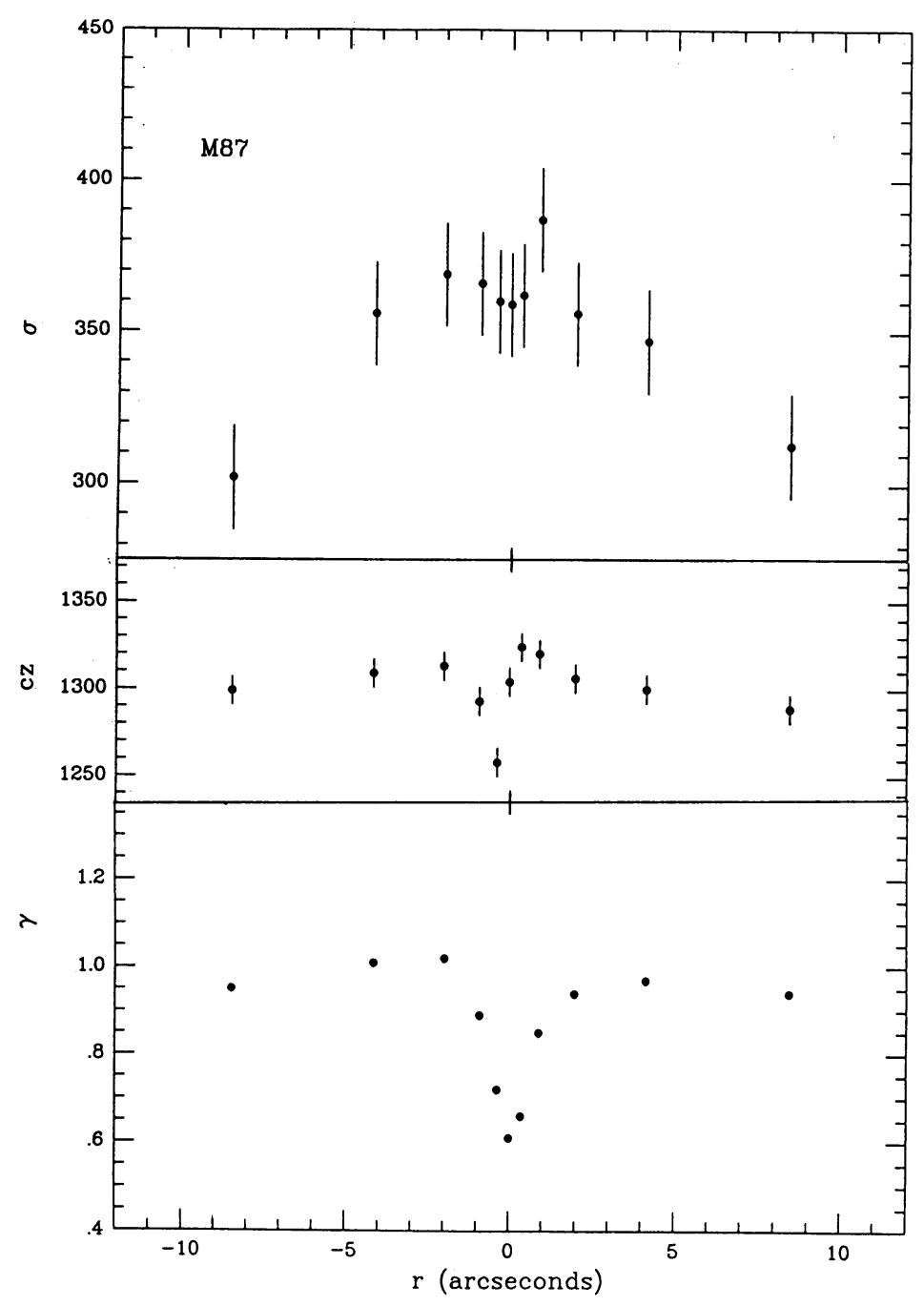

Figure 2. The run of velocity dispersion $\sigma$, rotation velocity $c z$, and line-strength $\gamma$, determined with the Fourier analysis program. The dispersion $\sigma$ does not rise within the inner 2 arcseconds, and the rotation velocity is small. Dilution by non-thermal light is indicated by the drop in $\gamma$ for $r<2$ arcseconds. 


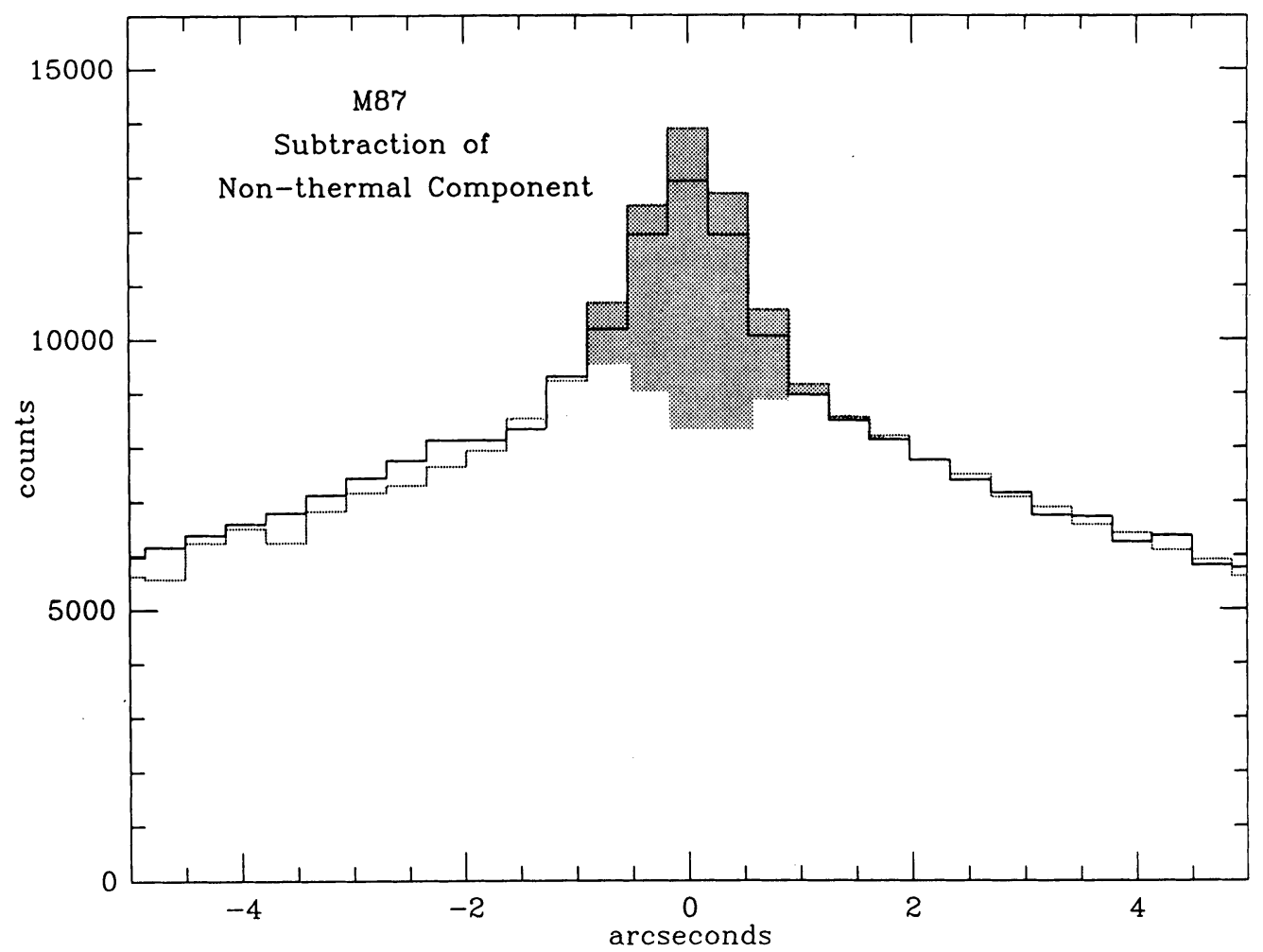

Figure 3. Profiles of M87 light entering the slit of the spectrograph for the two exposures of best seeing. The shaded area is that contributed by non-thermal light, as judged by the line-strength parameter $\boldsymbol{\gamma}$. 

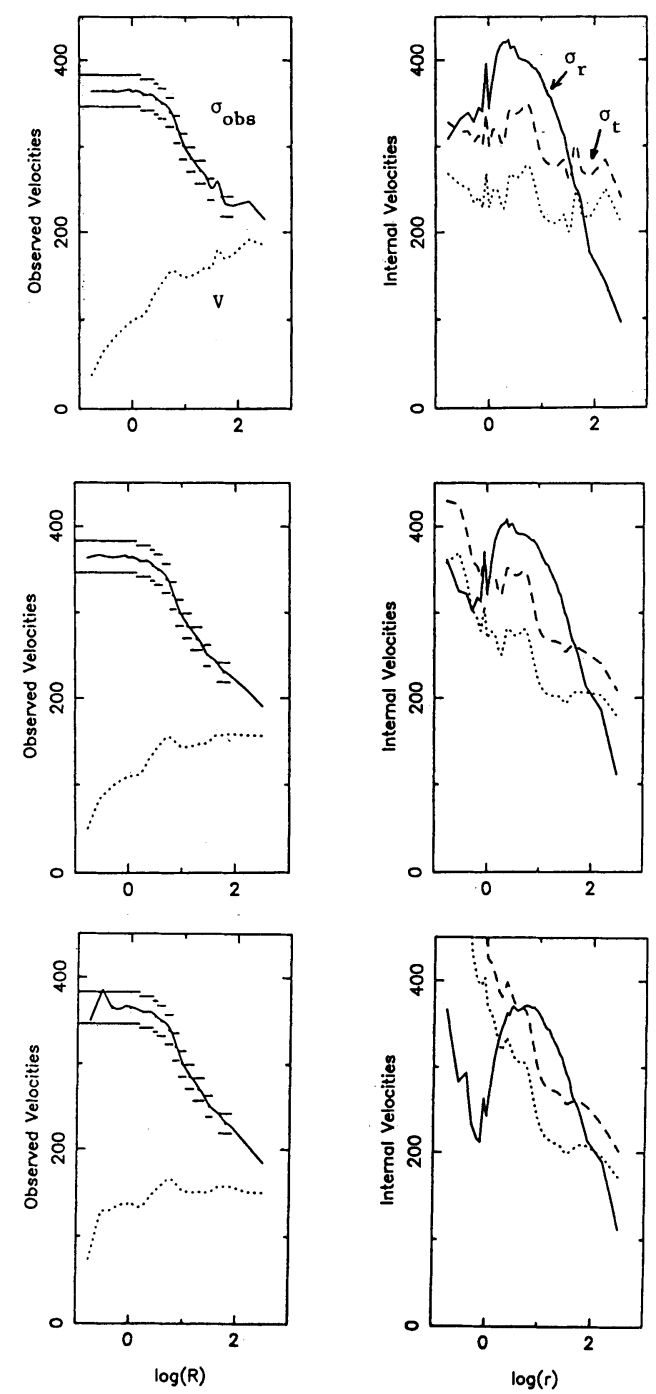

Figure 4. Models of M87. The right-hand plots show the model kinematic parameters. The left hand plots show projected "observables":

Predicted $\sigma$ is compared with the data; $V$ is the maxium rotation possible. The top panel is a constant $M / L$ model with no added black hole. The middle panel has a black hole of $10^{9} \mathrm{M}_{0}$, the bottom $3.6 \mathrm{x}$ $10^{9} M_{0}$. The latter is very anisotropic and predicts a high rotation rate which is not observed. 
consistent with the then-available data. From inspection of the distribution function for this constant $M / L$ model, it is clear that this model is not highly contrived. Merritt (1987) has questioned the stability of the Newton and Binney model, but Richstone (1988a) believes that the Richstone-Tremaine model should be more stable against bar-like instabilities because it is considerably less anisotropic.

Richstone and I have been working with my new data for M87 and we have been able to place tighter constraints on the mass of a putative black hole. The first point to make is that a rather mundane, constant $M / L$ model with no central black hole matches the data, as seen in Fig. 4. The model has a preference for radial orbits - the radial dispersion is about $20 \%$ larger than the tangential dispersion for $1 "<r<10 "$. There is nothing pathological or non-physical about this model, so we conclude that a central black hole is not required by the available data.

On the other hand, are the data consistent with a supermassive black hole? We find that a supermassive black hole $M<10^{9} M_{0}$ is tenable, because the observed velocity dispersion should not rise appreciably within $1 "$. In such a model, the tangential dispersion exceeds the radial dispersion for $r<1 "$, and if the angular momentum of the orbits is aligned, there would be a detectable rotation of about $V \sim 100 \mathrm{~km} / \mathrm{sec}$. However, the model is not very anisotropic, so random misalignment of the orbits is reasonable. In contrast, hiding a $3.6 \times 10^{9} \mathrm{M}_{0}$ black hole requires a tangential dispersion more than twice as large as the radial dispersion. These basically circular orbits would have to be turned by what Richstone calls "a Maxwell demon" so that their angular momentum cancel, otherwise there would be a significant rotation $V \sim 130 \mathrm{~km} / \mathrm{sec}$, completely incompatible with the observations. For masses greater than this the situation becomes rapidly worse; for $M>4 \times 10^{9} M_{0}$ the velocity dispersion measurements alone are sufficient to reject the model. The Sargent and Young model, in which a massive black hole of 3-5 $\times 10^{9} M_{0}$ was needed to account for the departures of the luminosity profile from a King law, is effectively ruled out.

In summary, we find: (1) a rising velocity dispersion in the core that levels out within $1 "$; (2) no significant rotation; (3) a central spike dominated by non-thermal light. No black hole is required to match these observations, but a black hole of $M<10^{9} M_{0}$ can be fitted in rather comfortably. To rule out masses less than this requires significantly higher resolution, like that available with HST. It is possible to make a model with a $3 \times 10^{9} M_{0}$ black hole which matches the data, but the model is contrived in the sense that the central stars are on rather circular orbits which run in opposite directions. It would be interesting to know if an accreting black hole would be able to hide itself by selectively depleting radial orbits, thus altering a previously isotropic distribution to make this unusual one. Richstone (1988b) believes that such a detailed question is beyond present modeling abilities. 


\section{SUPERMASSIVE BLACK HOLES IN M31 AND M32}

Most of us believe that M87 does have a supermassive black hole, and there is some evidence for it. Unfortunately, our prejudice and some supporting evidence does not constitute a proof. Richstone and I have repeatedly emphasized that demonstrating the failure of all reasonable models with constant $M / L$ is the minimum necessary step toward a proof. At this time, such a step cannot be taken for $M 87$, but it can be taken for M31 and M32 where the spatial resolution is some 20 times greater. Whereas contrived models are required support the idea of a a massive black hole in M87, on the contrary, contrived models are needed to avoid the conclusion that M31 and M32 contain dark, central mass concentrations.

Early observations of M31 by Lallemand et al. (1960) and of M32 by walker (1962) found rapid rotation in both systems down to a few arcseconds radius, about 10 pc. In 1974, Light et al. imaged M31 with the balloon-borne Stratoscope II and observed a central light spike that, unlike M87, is composed of stars. These early data hinted at the possibility of exotic kernels in both of these galaxies, the closest with large spheroidal components.

Tonry (1984) and Dressler (1984) obtained high S/N spectra of M32 which confirmed the rapid rotation down to small radii and showed an apparent rise in the velocity dispersion as well. Dressler (1984) found an even more dramatic signature of a dynamical subsystem in M31: the projected velocity dispersion and rotation rise abruptly to 230 $\mathrm{km} / \mathrm{sec}$ and $100 \mathrm{~km} / \mathrm{sec}$ respectively. Kormendy (1988b) obtained new spectroscopy for M31 in better seeing, which was matched by Dressler, who also obtained new data for M32 (Dressler and Richstone 1988), as did Tonry (1987).

All these data agree well. The interpretation is best handled, in my opinion, in the paper by Richstone and myself because we are the only ones to construct dynamical models of a general nature. We have used a maximum-entropy version of the Richstone-Tremaine program and have developed a new way to compare model predictions with the data. Rather than attempting to deconvolve data to compare with theoretical models, we have written a computer program that turns models into synthetic spectra. With the model predictions of projected velocity dispersion and rotation velocity, we synthesize the spectrum of the galaxy at a series of radial positions. This synthesis automatically folds in the seeing profile and the slit dimensions. These spectra are subjected to Fourier analysis, just like the real data, thus removing all of the uncertainties of comparing model predictions to observations.

We were unable to construct constant $M / L$ models for either $M 31$ or M32. The situation is quite different from the M87 case because of the strong rotation signature which limits the choice of orbits. For example, the peak in the dispersion profiles can be due to either a true dispersion or a very steep rotation gradient. The latter directly implies a rising $M / L$ and the former can be modeled with constant $M / L$ only if the central orbits are radial, which contradicts the observed rotation rate. We conclude that approximately $8 \times 10^{6} M_{0}$ of dark 
material are needed in the central $100 \mathrm{pc}^{3}$ of $\mathrm{M} 32$, and about 3-7 $\mathrm{x}$ $10^{7} M_{0}$ in $M 31$. This high density of $\rho>10^{5} M_{0}$ per $p c^{3}$

effectively rules out all but a black hole or a cluster of dense objects like neutron stars or white dwarfs. Goodman and Lee (1988) have studied the latter possibility and conclude that it is tenable, but that the effective radii of such systems would have to be larger than 0.1 arcseconds. Thus, to the degree that we are willing to contemplate a cluster of many million neutron stars in a 1 pc $^{3}$ volume, the case for a black hole must await HST observations to rule out this exotic possibility.

Recently, Gerhard (1988) has suggested another alternative to a supermassive black hole in M31, that of a stellar bar of dimension $\sim 1$ pC that happens to be pointing along our line of sight. Apparently such things are possible, but are not known to exist and are wholly unlike the kiloparsec-long bars Gerhard compares them to. Gerhard is partly motivated by Kormendy's (1988b) claim that the very center of M31 is disk-like. Richstone and I are unconvinced of this because: (1) my data do not confirm the drop in $\sigma$ at $r=2$ " that Kormendy sees; (2) even if the dispersion drops at $2 "$ ", this may not be relevant to the much smaller scale of the black hole; and (3) the Stratoscope II imaging shows no sign of a flattened system. Gerhard is betting that Kormendy is right about the nuclear disk. Even if he is, the mini-bar model is ad hoc, and contrived and unlikely, in my opinion. As yet, Gerhard has not actually modeled the distribution function, projection effects, or seeing corrections, so it is not even clear that the model can quantitatively reproduce the observations.

In summary, while an ironclad case for supermassive black holes cannot yet be made for M31 and M32, the alternatives are very exotic. As opposed to the M87 case, one is hard pressed to come up with a model that does not have a large amount of dark mass in a small volume.

\section{OTHER CASES}

Though M31, M32, and M87 are the best studied cases and have received the most attention, important data for other galaxies are now becoming available. Kormendy (1988c) and Jarvis and Dubath 1988) have obtained spectra for the very center of the Sombrero galaxy, N4594. Kormendy finds both kinematic and photometric evidence for a nuclear disk rotating rapidly at $V=230 \mathrm{~km} / \mathrm{sec}$. Like in the cases of $M 31$ and $M 32$, the rapid rotation severly constrains anisotropic models, with the result that no constant $M / L$ models are possible. Kormendy concludes that $a \sim 10^{9} \quad M_{0}$ object is the most reasonable. On the face of it, this is the best case yet for a supermassive black hole. However, because this galaxy is at a distance of nearly $20 \mathrm{Mpc}$, the volume constraint is some $10^{4}$ times weaker than in M31 or M32. That is, any dark system with a density $\rho \geqslant 10^{3} M_{0}$ per $p c^{3}$ will serve, so it is much more difficult to rule out dense star clusters. Again, however, they must be rather dark, and the change in stellar population, as Kormendy points out, is uncomfortably abrupt. 
Kormendy (1987) also reports a similar steep rotation gradient in NGC 3115, which could signal the presence of a black hole of $5 \times 10^{8}$ $M_{0}$. These data were obtained in rather poor seeing, so conclusions are tentative.

Keel, at this conference, has reported kinematic data for M81 that imply an upper limit of $2 \times 10^{7} M_{0}$ for any central mass. He bases his analysis on a scaled up version of the M31 models by Dressler and Richstone.

I only have time to mention very important new work on discrete kinematical subsytems in the cores of galaxies. The discovery of a counter-rotating core for IC 1459 by Franx and Illingworth (1988) has spurred high resolution kinematic studies of other early type galaxies. Though not a common phenomenon, cases of high rotation and dispersion in the nucleus, not always aligned with the major axis of the host galaxy, are proving surprisingly easy to find (Jedrzejewski and Schechter 1988, Bender 1988). A popular interpretation of such discrete subsystems is that they are merger products, but it is possible that some may reveal the existence of supermassive black holes. The key datum in this respect is whether the kinematic structure has been spatially resolved, for example, whether the rotation curve reaches its maximum outside the seeing disk. In resolved cases like IC 1459 the merger remnant interpretation is particularly appealing since constant $M / L$ models can easily be fashioned. Some of the cases may not be spatially resolved, so higher resolution spectra are vitally needed in order to look for the signatures of supermassive black holes.

There is quite a bit of circumstantial evidence that our own Galaxy harbors a supermassive black hole, much of it having to do with providing an energy source for the activity seen in the Galactic center. However, there is some kinematic evidence as well that 1-4 $x$ $10^{6} M_{0}$ are concentrated within the central few parsecs, as reviewed by Genzel and Townes (1987). Serabyn and Lacy (1985) trace the loci of several ionized gas streamers and model these as orbits around $3.3 \mathrm{x}$ $10^{6} M_{0}$ contained within $R<0.5 \mathrm{pc}$. If true, this is a constraint on density much like that for M31 and M32. The uncertainty in these kinds of analyses is the assumption that the virial theorem can be applied or that the gas is moving in simple orbits. For example, Geballe et al. (1984) have discussed the possibility that the gas is outflowing. Motions of stars are still the best way of uncovering a black hole, but such measurements are still inconclusive for the Galactic center (e.g., Sellgren et al. 1987). The case for a supermassive black hole in our own Galaxy is tantalizing, but the evidence is well short of a proof. Perhaps the most interesting conclusion so far is that the putative black hole is at least an order-of-magnitude less massive than the one in M31.

Since the work by Young, Sargent, and collaborators, it has become increasingly apparent that there are many other galaxies like M87 for which King models fail to describe the core structure, as discussed by Lauer (1985) and Kormendy (1985). For some of these systems the unusual light distributions could signal the presence of supermassive black holes, but there are few kinematic data and, in 
general, their greater distances make detection of black holes more difficult. HST observations will make a crucial contribution by providing high resolution spectroscopy for these systems.

Finally, there is a good deal of circumstantial evidence that massive black holes are present in lower luminosity AGNs, as reviewed by Filippenko (1988). This is evidence of a quite different sort than the stellar dynamical data I have been presenting, so I will omit a review of it here.

\section{IMPLICATIONS}

In my opinion, the observational evidence for supermassive black holes is as follows. M31 and M32 are two cases for which detection of a black hole is probable. Models without a dark, central mass concentration are contrived, and the alternative to a supermassive black hole, a dense cluster of collapsed stars, is certainly no less exotic. HST observations will be decisive in these cases. M87, N4594, N3115, and the Milky Way are cases for which detection of a massive black hole is possible, but for each case there is an acceptable model without a black hole. Excluding our own Galaxy, HST observations could promote these cases from the possible to the probable. (Of course, "probable" examples will be all the more important if some cases are considered certain.) Photometric and kinematic data for some half-a-dozen other cases is suggestive, but insufficient for serious modeling. We are making progress towards proving our favored hypothesis that a supermassive black hole is, indeed, the core of an AGN engine.

From the most conservative point of view, there is no observational evidence against supermassive black holes! Goodman and Lee (1988) and Rees (1988) have suggested that if these massive black holes are present, very luminous flares should be observed when the occasional star is swallowed. However, such an event might only be observable for a few years every 1000 years or so, thus the observational constraint may be quite weak.

My conclusions, if correct, have further implications:

(1) It would appear that supermassive black holes are relatively common in galaxies, suggesting that most galaxies went through an AGN period (or are failed AGNs). This argues against the model in which rare galaxies are active for a significant fraction of their lifetimes and suggests instead that most galaxies experienced a brief period of activity. Cavaliere et al. (1988) discuss this issue in detail.

(2) The largest black holes might have masses less than $10^{9} M_{0}$. A massive black hole could be the offspring of the spheroidal component of a galaxy, thus late-type spirals and irregulars, about half of all galaxies, may not have one. There may be a rough scaling of black hole mass with the mass of the spheroidal component, as suggested by the sequence M87, N4594, M31, M32, and the Milky Way. M32 does not now have a larger spheroidal mass than the Milky Way, but it is thought 
that $M 32$ has been tidally stripped to a significant extent.

(3) If galaxies like M31 and M32 with little or no activity in their nuclei harbor supermassive black holes, an efficient cleaning mechanism must be at work to prevent capture by the black hole of gas lost from evolving stars. The energy requirements for a galactic wind, for example, are modest.

\section{CONCLUSION}

Though we have not taken the final step in proving the existence of supermassive black holes, the past decade has been an extremely productive one towards that end. We have improved our observational skills and have honed theoretical tools on promising cases. As we wait for the successful launch of the Hubble Space Telescope, we are well placed to make observations that should demonstrate the reality and prevalence of this remarkable phenomenon.

\section{REFERENCES}

Bender, R. 1988, preprint.

Binney, J., and Mamon, G. A. 1982, M. N. R. A. S., 200, 361.

Cavaliere, A., Giallongo, E., Padovani, P., and Vagnetti, F. 1988, preprint.

Dressler, A. 1980, Ap. J. Lett., 240, L11.

Dressler, A. 1984, Ap. J., 286, 97.

Dressler, A., and Richstone, D. 0. 1988, Ap. J., 324, 701.

Duncan, M. J., and Wheeler, J. C. 1980, Ap. J. Lett., 237, L27.

Filippenko, A. V. 1988, in Supermassive Black Holes, ed. M. Kafatos, (Cambridge, Cambridge University Press), pp. 104-119.

Franx, M., and Illingworth, G. D. 1988, Ap. J. Lett., 327, L55.

Geballe, T. R., et al., 1984, Ap. J., 284, $1 \overline{18}$.

Genze1, R., and Townes, C. H. 1987, Ann. Rev. Astr. Ap., 25, 377.

Gerhard, 0. E., 1988, M. N. R. A. S. 232, 13p.

Goodman, J., and Lee, $\bar{H} . \bar{M} . \overline{19} 8 \overline{8}$, preprint.

Jarvis, B. J., and Dubath, P. 1988, Astr. Ap., in press.

Jedrzejewski, R., and Schechter, P. L. 1988, Ap. J. Lett., 330, L87. Kormendy, J. 1985, Ap. J. Lett., 292, L9.

Kormendy, J. 1987, in IAU Symposium 127, in Structure and Dynamics of Elliptical Galaxies, ed. T. P. De Zeeuw, (Dordrecht: Reidel), pp. 17-36.

Kormendy, J. 1988a, private communication.

Kormendy, J. 1988b, Ap. J., 325, 128.

Kormendy, J. 1988c, preprint.

Lallemand, A., Duchesne, M., and Walker, M. F. 1960, Pub. A. S. P., $72,76$.

Lauer, T. R. 1985, Ap. J., 292, 104.

Light, E. S., Danielson, R. E., and Schwarzschild, M. 1974, Ap. J., $194,257$. 
Lynden-Be11, D. 1969, Nature, 223, 690.

Merritt, D. 1987, Ap. J., 319, 1.

Newton, A., and Binney, J. 1984, M. N. R. A. S., 210, 711.

Rees, M. J. 1984, Ann. Rev. Astr. Ap., $22, \overline{4} 7 \overline{1}$

Rees, M. J. 1988, Nature, 333, 523.

Richstone, D. 0. 1988a, in Supermassive Black Holes, ed. M. Kafatos,

(Cambridge, Cambridge University Press), pp. 87-97.

Richstone, D. 0. 1988b, private communication.

Richstone, D. 0., and Tremaine, S. 1984, Ap. J., 286, 27.

Richstone, D. 0., and Tremaine, S. 1985, $\frac{A p .}{J_{0}}, 296,370$.

Sargent, W. L. W., Young, P. J., Boksenberg, A., Shortridge, K., Lynds, C. R., Hardwick, F. D. A. 1978, Ap. J., 221, 731.

Sellgren, K., Hall, D. N. B., Kleinmann, S. G., Scoville, N. Z. 1987, Ap. J., 317, 881 .

Serabyn, $\bar{E}_{.}$, and Lacy, J. H. 1985, Ap. J., 293, 445.

Tonry, J. L. 1984, Ap. J. Lett., 283, L27.

Tonry, J. L. 1987, Ap. J., 322, 932.

Walker, M. F., 1962, Ap. J., 136, 695.

Young, P. J. 1980a, Ap. J.' 217, 287.

Young, P. J. 1980b, preprint.

Young, P. J., Westphal, J. A., Kristian, J., and Wilson, C. P., and Landauer, F. P. 1978, Ap. J., 221, 721. 


\section{DISCUSSION}

DIAZ Regarding the dilution of the visible absorption lines by a non-thermal continuum that you mentioned for M87, I would like to point out that a featureless continuum in the visible does not necessarily have to be of non-thermal origin. Indeed, the spectrum of a very young star cluster is also featureless in the visible but shows no dilution in the $\mathrm{Ca} I \mathrm{l}$ triplet lines if they come from the red supergiants associated with the cluster. Have you looked at the Ca II triplet in M 87?

DRESSLER I do have some Ca II triplet data, but have not yet looked at the line dilution. Your point is a good one, and I do not think that any of my present data on M 87 can rule out the possibility that some of the featureless continuum might come from young stars.

DE ROBERTIS In your nuclear spectrum of $M 87$, there was substantial, broad [N I] $\lambda 5200$ emission. Proper subtraction is crucial to your $\mathrm{Mg}$ velocity dispersion. Can you comment on how you did this?

DRESSLER I made certain that the continuum fitting program was not fooled by the emission-line features, including [N I]. I also tested the procedure by adding an artificially broadened spectrum to the non-thermal component, and verified that the Fourier program got the right answer, in spite of the contamination.

GASKELL What about $M$ 33? There is evidence for quasar activity in it (e.g., a compact strong variable $X$-ray source). Also, earlier in the week we heard about a number of $\mathrm{Sc} / \mathrm{Sd}$ galaxies with quasar activity.

DRESSLER Late-type spirals have been little studied. I know of no kinematic data suggestive of supermassive black holes in any. Velocity dispersions, by the way, are not readily obtained in spectra with young stars, but rotation curves are tractable. Galaxy classification is difficult at high redshift, so ascribing late-types to host galaxies may be questionable.

MARSHALL It is certainly important to constrain the masses of black holes because as physical interpretations of pure luminosity evolution argue for large $M$ (> 10 $10-11 M_{\odot}$ ) in AGNs at or near the present epoch. I suggest that the kinematic data cannot yet rule out this possibility for AGNs because it is expected that the galaxy would still be active, making the observation very difficult.

DRESSLER You make a good point, that we could be selecting against extremely massive black holes because of their continuing activity. Also, they could be quite rare, and obviously we have not sampled very deeply.

SIMKIN Can you constrain your results in $M 31$ by using the gas velocity information in Rubin's echelle spectra which show that the $\mathrm{H} \alpha$ in the minor axis breaks up into discrete velocity filaments?

DRESSLER I am only aware of the paper in A.J. this year that shows a complex system of ionized gas in the bulge of M 31. These data, however, refer to a larger scale of $10^{\prime \prime}-100^{\prime \prime}$. 